\title{
The superlattices of discrete breathers in the 1D crystal model
}

\author{
D.V. Laptev \\ laptev.denis@mail.ru
}

B.I. Verkin Institute for Low Temperature Physics and Engineering, NAS of Ukraine, 47 Lenin Ave., Kharkov 61103

The dynamics of the discrete breather superlattices in the model of the $1 \mathrm{D}$ anharmonic Hirota lattice is considered. The analogues of the discrete breather for the finite-size system with periodic boundary conditions are presented. For the analogue of the discrete breather for the finite-size system the stability and the effect of dissipation on the dynamics are discussed. Using the exact solutions of the Hirota lattice equation in the form of discrete breather superlattices the asymptotic interaction energy between two breathers is investigated. It is shown that for the considered parameters of the solutions discrete breathers in the superlattice of type I repel, while in the superlattice of type II attract each other.

Keywords: discrete breather, intergable models, breather superlattices.

\section{The 1D Hirota lattice model.}

The concept of discrete breathers has become the one of the most intriguing topics in the solid state physics. Discrete breathers (DB) or localized anharmonic vibrations (LAV) are the dynamically stable spatially localized and periodic in time nonlinear excitations of the lattice systems $[1,2]$. DBs play an important role in the nonlinear dynamics of the lattices, conducting properties, phase transitions and catalysis processes in solids. During the last decade a great amount of numerical investigations on DB using molecular dynamics (MD) method and density function theory (DFT) in the models of different materials e.g. metals, ionic crystals, graphene and graphane, carbon nanotubes and other materials has been made [1-6]. In the review [7] the results on gap DBs in two- and three-dimensional crystals have been summarized. In [8] the molecular-dynamics simulations of DBs in the crystals with $\mathrm{NaCl}$ structure with different ratios of atomic masses of components have been presented.

In this paper the properties of single DBs and the superlattices of DBs in the exactly intagrable model of the anharmonic atomic chain - Hirota lattice - is considered [9-15]. The exactly integrable models of the lattice systems are important because for these models it is often possible to obtain the analytic expressions for the lattice excitations and for their main physical characteristics such as energy, momentum, etc. The most studied integrable lattices are the Toda lattice [16] and the system of Ablowitz-Ladik [17].

Hirota lattice is a model of the one-dimensional anharmonic chain of atoms, for which only the nearest neighbors' interaction is taken into account $[10,11]$.

$$
\begin{aligned}
\frac{m \ddot{u}_{n}}{1+\frac{\pi^{2}}{4} \frac{\dot{u}_{n}^{2}}{s^{2}}}=\frac{2}{\pi} \gamma d_{0}\{ & \tan \left[\frac{\pi}{2}\left(\frac{u_{n-1}-u_{n}}{d_{0}}\right)\right]- \\
& \left.-\tan \left[\frac{\pi}{2}\left(\frac{u_{n}-u_{n+1}}{d_{0}}\right)\right]\right\},
\end{aligned}
$$

where $u_{n}$ is the displacement of the $n$-th atom in the chain. The kinetic term of (1) is a similar as one for the modified discrete sine-Gordon model [18]. In the right side there are the nonlinear (tangential) interaction forces between the nearest neighbors. Equation (1) is equivalent to the exactly integrable system of the nonlinear self-dual network (NSDN) equations $[9,19]$ for currents strengths $\left(I_{n}\right)$ and voltages $\left(V_{n}\right)$ describing the transmission line with the nonlinear inductances $L\left(I_{n}\right)$ and capacitances $C\left(V_{n}\right)$.

$$
\frac{d}{d t}\left[C\left(V_{n}\right) V_{n}\right]=I_{n}-I_{n+1}, \quad \frac{d}{d t}\left[L\left(I_{n}\right) I_{n}\right]=V_{n-1}-V_{n},
$$

where

$$
C\left(V_{n}\right)=V_{n}^{-1} \arctan V_{n}, \quad L\left(I_{n}\right)=I_{n}^{-1} \arctan I_{n} .
$$

The NSDN equations are the nonlinear telegraph equations.

In the dimensionless coordinates

$$
\frac{u_{n}}{2 d_{0} / \pi}=\phi_{n}, \frac{\tau}{\left(d_{0} / s\right)}=t
$$

the Hirota lattice equation has the form:

$$
\frac{\ddot{\phi}_{n}}{1+\dot{\phi}_{n}^{2}}=\tan \left(\phi_{n-1}-\phi_{n}\right)-\tan \left(\phi_{n}-\phi_{n+1}\right) \text {. }
$$

The integral of motion energy corresponding to the equation (5) has the form $[10,11]$ :

$$
E=\frac{1}{2} \sum_{n=-\infty}^{+\infty} \ln \left(1+\dot{\phi}_{n}^{2}\right)-\frac{1}{2} \sum_{n=-\infty}^{+\infty} \ln \left[\cos ^{2}\left(\phi_{n}-\phi_{n-1}\right)\right] .
$$

For small values of the displacements and velocities of atoms the Hirota lattice model reduces to the $\beta$-Fermi-PastaUlam ( $\beta$-FPU) lattice model [20]. Equation (5) is equivalent to the discrete modified Korteweg - de Vries (dmKdV) equation [9]. In the long-wave limit equation (5) reduces to the continuous modified Korteweg - de Vries (mKdV) equation [9].

R. Hirota [9] has shown that the system of NSDN equations (2), (3) and equation (5) are exactly integrable and 
has found their multi-soliton solutions. M.M. Bogdan [10] obtained the moving and standing discrete breather solutions for the equation (5) for the first time. In the dimensionless coordinates the standing DB solution of the equation (5) has the form

$$
\phi_{n}^{(b)}(t)=(-1)^{\mathrm{n}} \arctan \left[\sinh (\kappa / 2) \frac{\cos \omega t}{\cosh \kappa n}\right] .
$$

The cyclic frequency of oscillations equals

$$
\omega= \pm 2 \cosh (\kappa / 2) \text {. }
$$

The DB solution of the equation (5) has the similar structure as the breather of sine-Gordon equation. Zhou et al. [21] have obtained the discrete breather solution (7) of the equation (5) using the wronskian technique. The expression (7) is the solution of equation (5) for the infinite lattice. It can be used for description of the highly localized excitations far from the boundaries in the finite-sized lattices. The energy of discrete breather $(7)$ in the infinite chain equals $[6,7]$ :

$$
E_{b}=\kappa \text {. }
$$

The generalized models of the $1 \mathrm{D}$ anharmonic crystal and equivalent nonlinear transmission line contain the terms corresponding to the dissipation processes and the action of the external forces [22]. The equation for the generalized 1D Hirota lattice model has the form:

$$
\begin{aligned}
& \frac{m \ddot{u}_{n}}{1+\frac{\pi^{2}}{4} \frac{\dot{u}_{n}^{2}}{s^{2}}}=\frac{2 \gamma d_{0}}{\pi} \tan \left[\frac{\pi}{2}\left(\frac{u_{n-1}-u_{n}}{d_{0}}\right)\right]- \\
& -\frac{2 \gamma d_{0}}{\pi} \tan \left[\frac{\pi}{2}\left(\frac{u_{n}-u_{n+1}}{d_{0}}\right)\right]-\frac{\pi}{2} \lambda \frac{\dot{u}_{n}}{s}+f_{n}^{(e x t)}(t),
\end{aligned}
$$

where $\lambda$ is the dissipation constant, $f_{n}^{(e x t)}(t)$ is the external varying in time force. The generalized system of the NSDN equations describing the equivalent nonlinear transmission line in dimensionless units has the form [22]:

$$
\begin{aligned}
& \frac{d}{d t}\left[C\left(V_{n}\right) V_{n}\right]+G V_{n}=I_{n}-I_{n+1}, \\
& \frac{d}{d t}\left[L\left(I_{n}\right) I_{n}\right]+R I_{n}=V_{n-1}-V_{n}+E_{n}(t),
\end{aligned}
$$

where $C\left(V_{n}\right), L\left(I_{n}\right)$ are determined by equation (3), $G$ is the conductivity of the nonideal insulator, $R$ is the active resistance of the wires and $E_{n}(t)$ is the external electromotive force. In [23] the periodic vibrations that represent the symmetry-determined nonlinear normal modes have been investigated using the group-theoretical method in the LCand LCR-transmission lines.

\section{The superlattices of the discrete breathers and shock waves}

In [22] the new classes of periodic solutions expressed in terms of the Jacobi elliptic functions have been obtained for the Hirota lattice model and equivalent system of NSDN equations.

The obtained solutions are the spatially periodic waves describing the discrete breather and shock wave superlattices in the infinite lattice and in the lattice with arbitrary finite number $N$ of sites with the periodic

and zero-fixed

$$
\phi_{n+N}(t)=\phi_{n}(t)
$$

$$
\phi_{1}(t)=\phi_{N}(t)=0
$$

boundary conditions. In the small-amplitude limit these solutions reduce to the linear running and standing waves and in the essentially nonlinear limit to the separated discrete breathers or one-parametric solitons (kinks and antikinks).

A.S. Kovalev [24] has obtained the similar periodic solutions for the sine-Gordon equation. In [25] the breather lattice solution of the sine-Gordon equation has been investigated. In [26] the exact periodic solutions of the positive and negative modified Korteweg-de Vries equations have been found.

For brevity we will use the following notations: $\operatorname{sn}(\theta, \chi)$, $\operatorname{cn}(\theta, \chi), \operatorname{dn}(\theta, \chi)$ are the Jacobi elliptic functions, $\chi, \mu$ are the modules of the elliptic functions, $\chi^{\prime}=\sqrt{1-\chi^{2}}, \mu^{\prime}=\sqrt{1-\mu^{2}}$ are the additional modules of the elliptic functions.

The standing discrete breather superlattice of type I solution of the equation (5) has the form (Fig.1).

$$
\phi_{n}^{(S L I)}(t)=(-1)^{n} \arctan \left[A_{\mathrm{I}} \operatorname{sn}(b t, \chi) \operatorname{dn}(\kappa n, \mu)\right],
$$

where

$$
\begin{aligned}
& b= \pm \frac{2 \operatorname{dn}(\kappa / 2, \mu)}{1-A^{2} \operatorname{dn}^{2}(\kappa / 2, \mu)}, \\
& A_{\mathrm{I}}=\sqrt{\frac{\chi}{\mu^{\prime}}}=\frac{\operatorname{sn}(\kappa / 2, \mu)}{\operatorname{cn}(\kappa / 2, \mu)} .
\end{aligned}
$$

It is easy to see that the time and spatial periods of the superlattice of type I equals respectively

$$
T_{\mathrm{I}}=\frac{4 \mathbb{K}(\chi)}{b}, \quad \Lambda_{\mathrm{I}}=\frac{2 \mathbb{K}(\mu)}{\kappa} .
$$

Since the function $\operatorname{dn}(\theta, \mu) \neq 0, \forall \theta$ zero-fixed boundary conditions (13) are not satisfied for the solution (14).

Suppose that the length of the chain contains the integer number $M$ of the real spatial periods of the solution ( $M$ is a number of breathers in the superlattice). Periodic boundary condition (12) is satisfied if

$$
N=M \Lambda_{\mathrm{I}}=\frac{2 \mathbb{K}(\mu)}{\kappa} M .
$$

For $\mu \rightarrow 0$ the equations (14), (15) reduce to the solution describing the nonlinear homogeneous antiphase oscillations.

$$
\phi_{n}(t)=(-1)^{n} \arctan \left[\sqrt{\chi} \operatorname{sn}\left(\frac{2}{1-\chi} t, \chi\right)\right] \text {. }
$$

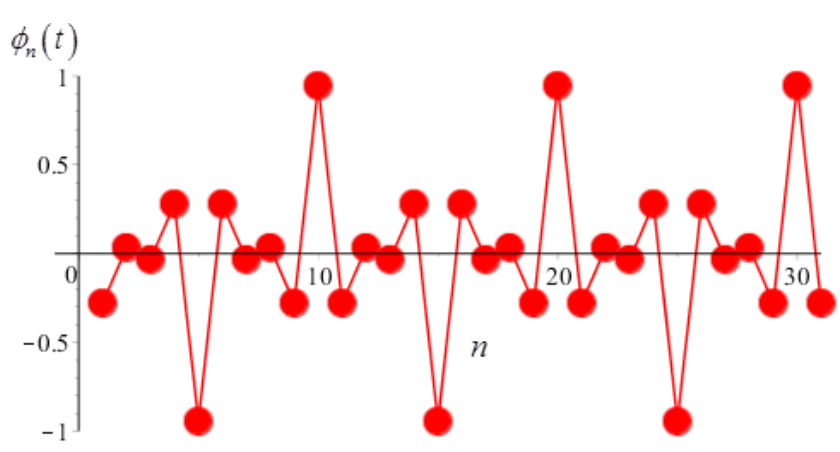

Fig. 1. Breather superlattice of type I. The number of sites in the lattice $N=30$, the number of breathers in the superlattice $M=6$, the value of the elliptic modulus $\mu=0.9999$ corresponds to the large separation of breathers in the superlattice. Time equals the quarter of period $t=T_{\mathrm{I}} / 4$. For the considered values of the parameters breathers oscillate in the opposite phase. 
For $\chi \rightarrow 0, \mu \rightarrow 1$ the solution (14), (15) reduces to the standing discrete breather. If $M=1$ expressions (14)-(17) represent the analog of discrete breather for the finite-sized lattice (Fig.2).

Using the Runge-Kutta method the linear stability of the analogue of discrete breather $(M=1)$ for the finite-sized lattice has been investigated. The evolution of the lattice was modelled using the initial conditions corresponding to the breather superlattice of type I (SLI) (14) with linear sinusoidal perturbation.

$$
\begin{gathered}
\phi_{n}(t=0)=\phi_{n}^{(S L I)}+0.01 \sin (n), \\
\frac{d}{d t} \phi_{n}(t=0)=\frac{d}{d t} \phi_{n}^{(S L I)} .
\end{gathered}
$$

The corresponding contour plot is shown in Fig.3. Simulations demonstrate the linear stability of the analogue of discrete breather.

The dynamics of the analog of the DB in the finite-sized lattice has been also modelled numerically (Fig.4). The dissipation coefficient of the equation (10) equals

$$
\lambda=0.1 \text {. }
$$

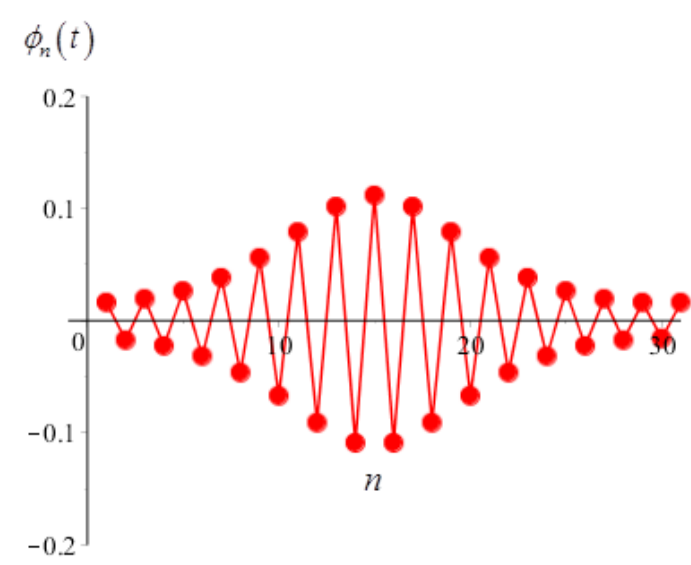

Fig. 2. Breather superlattice of type I containing one breather - the analogue of discrete breather for the finite-sized lattice. The number of sites in the lattice $N=30,, M=1, \mu=0.99, \mathrm{t}=3 T_{\mathrm{I}} / 4$.

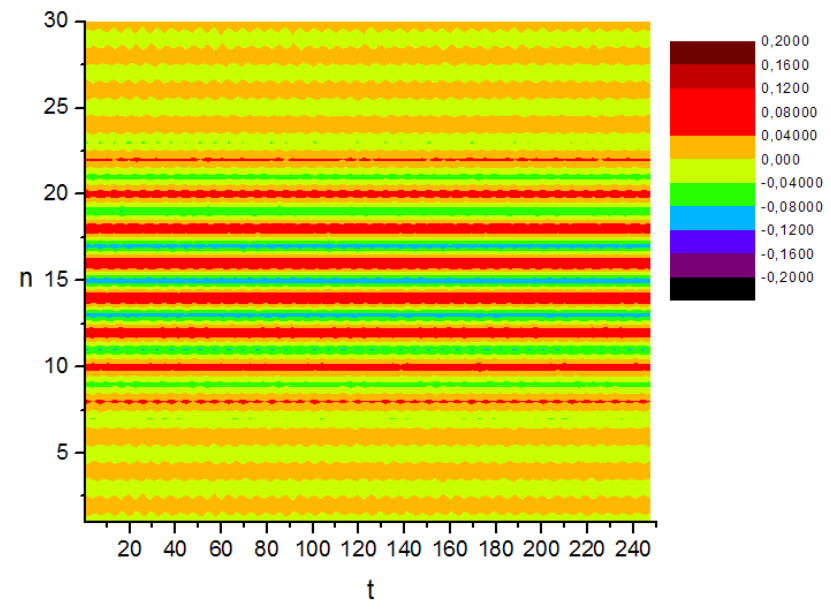

Fig. 3. (color online) The evolution of the analogue of discrete breather for the finite-sized lattice. A space time contour plot $\phi_{n}(t)$. Initial condition is the breather lattice solution of type I with linear sinusoidal perturbation. $N=30, M=6, m=0.99, \lambda=0, f_{n}^{(e x t)}(t)=0$, $t_{0}=T_{\mathrm{I}} / 4$. Time is measured in the periods of the oscillation. Boundary conditions are periodic.
From Fig 4 it is seen that nonlinear excitation loses energy due to the dissipation. The life-time of the DB analog is approximately 30 periods.

The standing discrete breather superlattice of type II has the form (Fig.5).

where

$$
\phi_{n}(t)=(-1)^{n} \arctan \left[A_{\mathrm{II}} \mathrm{cn}(\kappa n, \chi) \operatorname{cn}(q t, \mu)\right],
$$

$$
\begin{aligned}
& q= \pm 2 \mu^{\prime}\left(1+A_{\mathrm{II}}^{2}\right) \operatorname{cn}(a / 2, \chi), \\
& A_{\mathrm{II}}=\sqrt{\frac{\chi \mu}{\chi^{\prime} \mu^{\prime}}}=\frac{\sqrt{\chi \operatorname{sn}^{2}(a / 2, \chi)-\mu^{2}}}{\operatorname{cn}(a / 2, \chi) \mu^{\prime}} .
\end{aligned}
$$

It is easy to see that the time and spatial periods of the superlattice of type II equals respectively

$$
T_{\text {II }}=\frac{4 \mathbb{K}(\mu)}{q}, \quad \Lambda_{\text {II }}=\frac{4 \mathbb{K}(\chi)}{\kappa} .
$$

Zero-fixed boundary conditions (13) for the solution (22) can be satisfied if

$$
\begin{aligned}
\kappa & =\mathbb{K}(\chi)\left(2 l_{1}+1\right), \quad l_{1}=0,1,2, \ldots \\
\kappa N & =\mathbb{K}(\chi)\left(2 l_{2}+1\right), \quad l_{2}=1,2,3, \ldots
\end{aligned}
$$

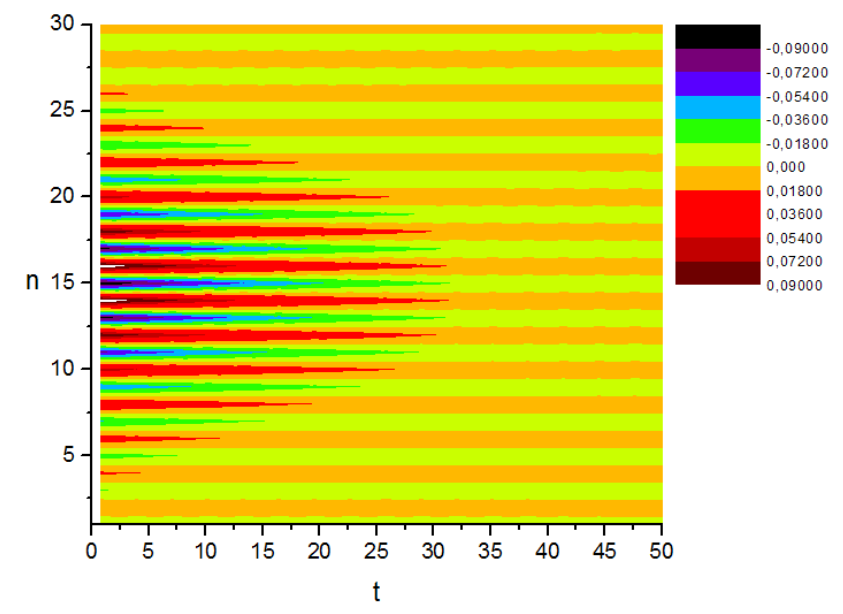

Fig. 4. (color online) The evolution of the analogue of discrete breather for the dissipative finite-sized lattice. A space time contour plot $\phi_{n}(t)$. Initial condition is the breather lattice solution of type I. $N=30, M=6, m=0.99, \lambda=0.1, f_{n}^{(e x t)}(t)=0, t_{0}=T_{\mathrm{I}} / 4$. Time is measured in the periods of the oscillation. Boundary conditions are periodic. Nonlinear excitation loses energy due to the dissipation.

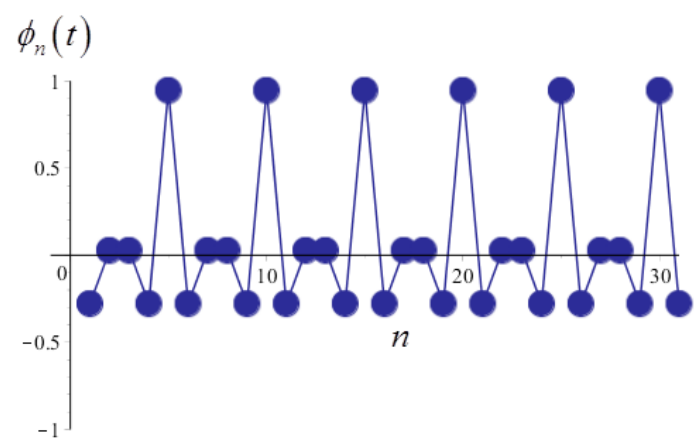

Fig. 5. Breather superlattice of type II. The number of sites in the lattice $N=30$, the number of breathers in the superlattice $M=6$, the value of the elliptic modulus $\mu=0.9999$ corresponds to the large separation of breathers in the superlattice. Time $t=0$. For the considered values of the parameters breathers oscillate in the same phase. 
Suppose that the length of the chain contains the integer number $M$ of the real spatial periods of the solution ( $M$ is a number of breathers in the superlattice). Periodic boundary condition (12) is satisfied if

$$
N=M \Lambda_{\text {II }}=\frac{4 \mathbb{K}(\chi)}{\kappa} M .
$$

For $\chi \rightarrow 1, \mu \rightarrow 0$ solution (22) reduces to the standing discrete breather.

The superlattices of type I and type II containing only two breathers each (Fig. 6) were considered and the energy of these superlattices were calculated.

By the definition of the integral of motion energy can be calculated for any arbitrary moment of time. Expression (28) is the energy (6) of the standing discrete breather superlattice of type I (14) in the chain with $N$ nodes for the moment of time $t=0$.

$$
E_{\mathrm{I}}=\frac{1}{2} \sum_{n=1}^{N} \ln \left[1+A_{\mathrm{I}}^{2} b^{2} \operatorname{dn}^{2}(\kappa n, \mu)\right] .
$$

The energy of the homogeneous oscillations (18) in the chain with $N$ nodes equals

$$
E_{0}=N \ln \frac{1+\chi}{1-\chi}
$$

The energy of the standing discrete breather superlattice of type II in the chain with $N$ nodes for the moment of time: $t=\mathbb{K}(\mu) / q$ equals

$$
E_{\mathrm{II}}=\frac{1}{2} \sum_{n=1}^{N} \ln \left[1+A^{2} \mu^{\prime 2} q^{2} \mathrm{cn}^{2}(\kappa n, \chi)\right] .
$$

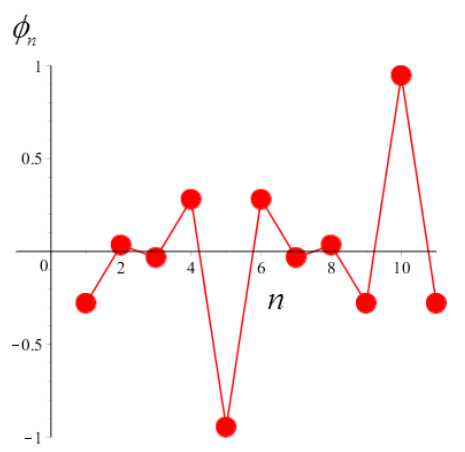

(a)

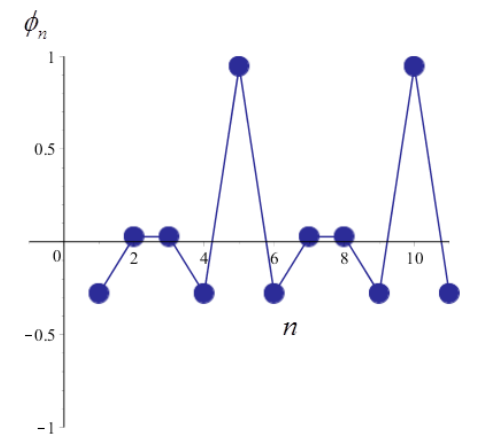

(b)

Fig. 6. Breather superlattices. The number of sites in the lattice $N=$ 10 , the number of breathers in the superlattice $M=2$, the value of the elliptic modulus $\mu=0.9999$ corresponds to the large separation of the breathers in the superlattice. (a) Breather superlattice of type $\mathrm{I}, t=T_{\mathrm{I}} / 4$, breathers oscillate in the opposite phase. (b) Breather superlattice of type II, $t=0$, breathers oscillate in the same phase.
In Fig. 7 the dependence of energy on parameter of the solution $\kappa$ for breather superlattices of type I and II (SL of type I, SL of type II), homogeneous anti-phase oscillation (HAO) and two free discrete breathers is shown.

It is seen, that for the chosen values of the solutions parameters the energy of superlattice of type I containing two breathers is larger then the energy of two free breathers, i.e. the energy of breathers' interaction in the SL of type I is positive. It means that the character of interaction of breathers oscillating in the opposite phase is repulsion. On the other hand breathers in the SL of type II oscillating in the same phase attract each other. For large values of the parameter $\kappa$ (large separation of breathers) the superlattices of both types reduce to two free discrete breathers.

In [22] the solution describing the moving discrete shock wave superlattice has been also found.

In [27] the results of the inelastic neutron measurements performed on the NaI crystals show the existence of the discrete breather superlattice under certain conditions.

\section{Conclusions}

The dynamics of the single discrete breathers and their superlattices of two types in the exactly integrable model of the 1D anharmonic crystal - the Hirota lattice model has been discussed. The periodic and zero-fixed boundary conditions have been considered for the arbitrary number of sites. The analog of the DB for the finite-size lattices has been presented. It has been shown that the analog of the $\mathrm{DB}$ for the finite-size lattice is linearly stable. Using the generalized Hirota lattice model the influence of dissipation on the breather dynamics has been discussed. It has been shown that the nonlinear excitation loses energy due to the dissipation. The life-time of the $\mathrm{DB}$ analog is approximately 30 periods. It was shown that for the considered parameters of the solutions DBs in the superlattice of type I repel, while in the superlattice of type II attract each other.

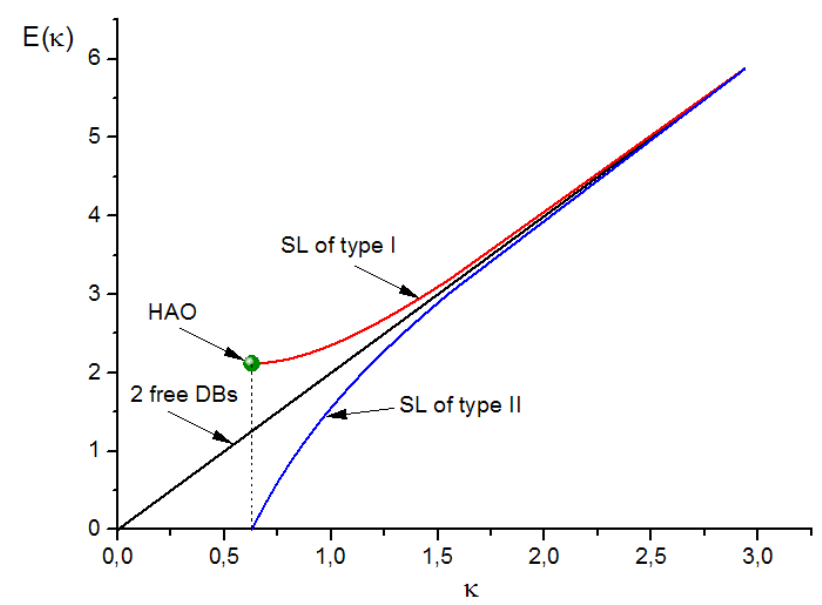

Fig. 7. (color online) The dependence of energy on parameter of the solution $\kappa$ for breather superlattices of type I and II (SL of type I, SL of type II) - upper red curve and lower blue curve respectively, homogeneous anti-phase oscillation (HAO) - green point from which the SL of type I starts and two free discrete breathers - middle black straight line. 
Acknowledgements. The author is grateful to professor M.M. Bogdan for helpful discussions during this research.

\section{References}

1. S. Flach, A. V. Gorbach, Phys. Rep. 467, 1 (2008).

2. D. K. Campbell, S. Flach, Yu. S. Kivshar, Phys. Today. 57, 43 (2004).

3. G. M. Chechin, S. V. Dmitriev, I. P. Lobzenko, D. S. Ryabov, Phys. Rev. B. 90, 045432 (2014).

4. G. M. Chechin, I. P. Lobzenko, Letters on materials. 4 (4), 226 (2014).

5. V.I. Dubinko, Letters on materials 4 (4), 283 (2014).

6. V.I. Dubinko, F. Piazza, Letters on materials 4 (4), 273 (2014).

7. S. V. Dmitriev, Letters on Materials. 1 (2), 78 (2011). (in Russian) [С. В. Дмитриев, Письма о материалах. 1 (2), 78 (2011).]

8. L.Z. Khadeeva, S. V. Dmitriev, Phys. Rev. B. 81, 214306 (2010).

9. R. Hirota, J. Phys. Soc. Jpn. 35, 289 (1973).

10. M.M. Bogdan, G.A. Maugin, Proc. Estonian Acad. Sci. Phys. Math. 52, 76 (2003).

11. M. M. Bogdan, D. V. Laptev, Uzhhorod University Scientific Herald. Series Physics. 24, 100 (2009). (in Ukrainian) [М.М. Богдан, Д. В. Лаптєв. Науковий вісник ужгородського університету. Серія Фізика. 24, 100 (2009).]
12. D. V. Laptev, Letters on Materials. 4 (4), 241 (2014).

13. D. V. Laptev, J. Phys. Soc. Jpn. 82, 014005 - 1 (2013).

14. D. V. Laptev, J. Phys. Soc. Jpn. 82, 044005 - 1 (2013).

15. M. M. Bogdan, D. V. Laptev, J. Phys. Soc. Jpn. 83, 064007 - 1 (2014).

16. M. Toda, Prog. Theor. Phys. Suppl. 45, 1974 (1970).

17. D. Cai, A. R. Bishop, N. Grønbech-Jensen, Phys. Rev. Lett. 72, 591, (1994).

18. W. J. Zakrzewski, Nonlinearity. 8, 517 (1995).

19. A. C. Scott, F. Y. F. Chu, D. W. McLaughlin, Proc. IEEE 61, 1443 (1973).

20. G. P. Berman, F. M. Izrailev, Chaos. 15, 015104 (2005).

21. J. Zhou, D. J. Zhang, S. L. Zhao, Phys. Lett. A 373, 3248 (2009).

22. D. V. Laptev, M. M. Bogdan, J. Math. Phys. 55, 042903 - 1 (2014).

23. G.M. Chechin, S.A. Shcherbinin, Communications in Nonlinear Science and Numerical Simulation. 22, 244 (2015).

24. A. S. Kovalev, Physics of the Solid State (Fizika Tverdogo Tela) 21, 1729 (1979). (in Russian) [А. С. Ковалёв, ФТТ 21, 1729 (1979).]

25. P. G. Kevrekidis, A. Saxena, A. R. Bishop, Phys. Rev. E 64, 026613 (2001).

26. P. G. Kevrekidis, A. Khare, A. Saxena, G. Herring, J. Phys. A: Math. Gen. 37, 10959 (2004).

27. M.E. Manley, D. L. Abernathy, N. I. Agladze, A. J. Sievers, Sci.Rep. 1, 4 (2011) . 